\title{
Calvarial Bone Segmentation from Medical Images by Image Processing Technique
}

\author{
K. Prahlad Rao \\ Asst. Prof., Dept. of Electrical and Computer Engg., King Abdulaziz University, Jeddah, Saudi Arabia
}

\begin{abstract}
In a given image, it is possible to extract only the required data from the intact image. This is known as image segmentation and is considered as one of the important image processing technique. Several algorithms do the segmentation on images but Watershed method, which is based on natural phenomenon of water flow in lakes, is attracting large number of researchers due to its fast action, simple algorithm to implement in computer and versatile for twin images too. The morphological watershed image segmentation is implemented on MRI images to extract calvarial bone (skullcap) from the scans. It is useful for evaluation of calvarial lesions, calvarial bone grafts and calvarial bone fractured patients that could help in diagnosis and treatments. The results are demonstrated and the method is explained. Applicability of the method for the calvaria related abnormalities are discussed.
\end{abstract}

Key words: Watershed image segmentation; calvarial bone; Brain; MR imaging; Matlab

\section{INTRODUCTION}

Image segmentation refers to partitioning of an image into distinct regions of interest that contain pixels with similar attributes. The main goal of segmentation is to differentiate the object of interest from the background of image. Thus the segmentation of image may be considered as an important step in image processing techniques. Several image segmentation techniques are being used for image analysis; some of them are general purpose while some are designed for specific images. There are several application and various algorithms for segmentation in image processing from general images to medical applications. But, there is no universal algorithm for segmentation of every medical image because each imaging system has its own specific limitations and the specific anatomical information required to diagnose the patient with different characteristics. A major difficulty of segmentation of medical images is the high variability in the images [1]. Different imaging modalities such as $\mathrm{x}$ ray, CT, MRI, ultrasound, PET, SPECT, etc., produces their own images [2]. Further to add, human anatomy itself shows major modes of variation. When these two prominent variations are considered together, analysis of the image, thus generally make high complexity and ambiguity. However, the computer advancements made it possible to implement image processing algorithms, including segmentation, on medical images in the digital domain at different accuracy and degree of complexity. Therefore, accurate segmentation of medical images becomes a key step in image processing which can help for suitable planning of diagnosis and treatment of the patient.

Human brain is the most complex organ of the body having various parts within it. Each part has distinct anatomical signature and functional characteristics which are all together encapsulated within a mechanically strong bony structure, known as the skull. The human skull bone is the most complex part that supports the face and teeth and thus forms a protective cavity for the brain, sensory organs, nerves and blood vessels from any mechanical damage but allows the passage of air and food [3]. Acting as a mechanical foundation, the skull may be divided into two groups: the cerebral cranium and the visceral cranium. The roof of the cranium is known as skullcap or calvaria, which is derived from the Latin word [4]. The calvaria could be affected by a variety of diseases. Congenital, traumatic, inflammatory, skull fractures and neoplastic lesions are the few to name related to calvarial lesions. Routine clinical examination may not be enough for any conclusive diagnosis of the disease in several occasions. Imaging plays an important role for the clinical diagnostic and therapeutic procedures. Radiologists prefer CT (computed Tomography) and MR imaging (Magnetic Resonance imaging) to scan head, but most of the findings from the scans do not exhibit distinctive imaging features in several cases [5][6][7]. The radiographic imaging varies according to their pathologic features. For evaluation of calvarial lesions from these images, the distinctive features are to be extracted which can guide the radiological diagnosis specifically. In this article, computer based algorithm is developed for segmentation of calvarial bone or skullcap alone from the medical images. This technique can guide the radiologists precisely for a specific diagnosis of lesions or delineating pathological regions, thus limiting the differential diagnosis.

\section{BACKGROUND}

Image segmentation may be described as the partitioning of an image into non-overlapping, constituent regions that are homogeneous with respect to some characteristics [8]. The procedure is one of the most critical and challenging task of image processing. It has various applications over a wide spectrum ranging from vehicle's number plate identification in traffic management to the cancer cell detection in clinical investigations. Several image segmentation techniques are available in literature, but there is no common method which can be considered good 
International Journal of Advanced Research in Computer and Communication Engineering

Vol. 4, Issue 12, December 2015

for all images [1]. As the number of algorithms developed is increasing each year continuously [9], it is considered as a great challenge by the computer vision researchers. Very popular image segmentation techniques that are still used today by the researchers are edge based [10], region based (11), histogram based [12], threshold based [13], model based, feature based and Watershed transformation which can be found in literature. Segmentation of medical images is relatively difficult but important part of image processing, thus become focus of attraction for the researchers from various fields.

Human body comprised of different organs and each organ consists of biological components such as bones, tissues and blood vessels. Each component of any organ can influence the health condition of the person. Medical images display the organs spatially by non-invasive method to view them for the characterization. X-ray CT and MRI are most widely used radiographic imaging techniques for clinical purposes. The scans from these systems are referred for quantification of tissue volumes, diagnosis, pathology, anatomical and functional information. Every scan of images differs in noise level, artifacts contrast and resolution when it is focused for a particular organ. For example, the most intricate, complicated and impressive organ is the brain which has different sections and is protected by the bone tissue (skull). Several research articles have been reported in the area of brain image segmentation for tumors, brain tissues (such as white matter, gray matter) and CSF (cerebrospinal fluid) from medical images based on various algorithms. It is equally an important objective to consider calvaria for segmentation which could help to understand the pathological changes in various forms of degenerative brain diseases. The segmentation of calvaria from medical images based on image processing algorithms did not come to the notice of the author from literature. Therefore, in this work the calvarial bone segmentation from Watershed algorithm is proposed which could help for clinical analysis of brain images.

\section{METHODOLOGY}

The human head mainly consists of brain and the outer bone. Below Fig 1 (A) (B) shows anatomy of the head and brain in sagittal and horizontal sections.

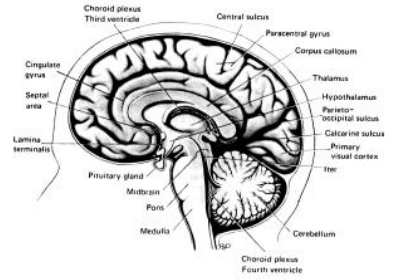

(A)

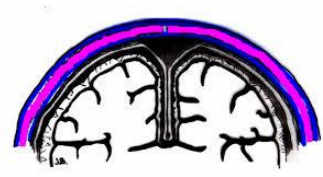

(B)
Fig. 1 Anatomy of Human head and Brain. (A) Sagittal plane cross section (B) Horizontal plane cross section

Human head shown in above figures may be considered as two major images are fused together in the form of brain tissue and the calvarial bone. The two layers are touching together and therefore, Watershed algorithm for segmentation is more suitable.

\section{Watershed Algorithm}

A. Principle

The principle of watershed transform suggests that any greytone image can be considered as a topographic surface. If this surface is flooded from its minima, and if the merging of water coming from different sources, similar to that water flowing from catchment area in a lake, then the image can be portioned into two different sets as the catchment basins and the watershed lines. For better understanding, one can imagine that the bird view over a large water source accumulating behind a storage dam. Suppose the transformation is applied to the image gradient, the catchment basins then theoretically correspond to the homogeneous grey level regions in the image. If a drop of water flowing the gradient of an image then, that drop flows a path through ultimately reach a local minimum. That means, by nature, the fluidic water always flows into the lower basin. In simple words, it is the line which determines where the drop of water will fall into the region. This classic algorithm was introduced by Luc Vincent and Pierre Soille [15], which was based on the concept of "immersion". This algorithm is particularly useful for segmenting objects that are touching one another. Initially, the concept was used by Digabel [14] which was later reviewed and implemented efficiently by Vincet [15][16]. The morphological watersheds provide a complementary approach to the segmentation of objects. It is useful in particular, for segmenting the objects which are touching each other. This algorithm being applied for medical image segmentation by various researchers [17][18].

\section{B. Proposal of the Work}

For implementing the watershed algorithm, generally, three methods are followed; distance transform approach, the gradient method and the marker controlled approach.

In the distance transform approach, the distance from every pixel to the nearest non-zero valued pixel is calculated. This is the first step of pre-processing the image before implementing the watershed algorithm. Important steps of the proposed work are shown in the below Fig. 2. In this work, T1 weighted MRI scans of the head and brain from the web source are taken as input images. To implement the algorithm, the computer program is written and executed in Matlab Software. Each step of the program is explained in detail in the following paragraphs.

The MRI scan of the brain with calvaria is considered as original input image. It is loaded in Matlab platform to read as input image for implementation of Watershed algorithm. Most of the medical images have inherent noise and with different contrast levels. They are pre-processed to remove the noise. This is carried out by subtraction of opened image from the original image. Then the correct level of threshold is calculated for a value by which calvarial bone can be segmented. By thresholding the image at this value, it is then converted to binary and its inversion form. For the binary image, distance is calculated all the nearest non-zero values are pushed within the image. 
International Journal of Advanced Research in Computer and Communication Engineering Vol. 4, Issue 12, December 2015

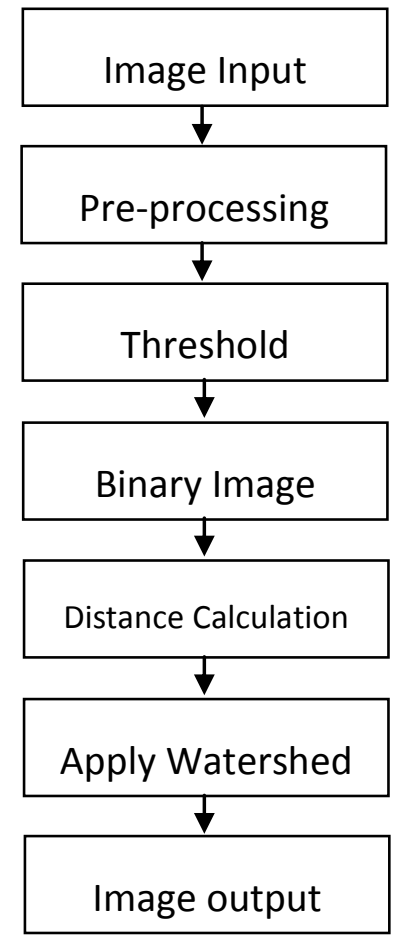

Fig. 2 Steps for Proposed Watershed Image Segmentation The purpose of doing this step is to force the local minima inside the image to facilitate the water filling within it, hence to apply the watershed. Finally, watershed is applied such that all the ridges are given zeros and the catchment basins with non-zeros.

\section{EXPERIMENTAL RESULTS AND DISCUSSION}

A set of two MRI scans of the brain with calvaria are taken as input images; one scan is from sagittal and another from horizontal plane cross sections. Both images are shown in the below Fig.3 as (A) and (B) respectively.

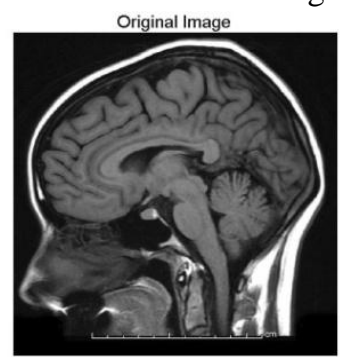

(A)

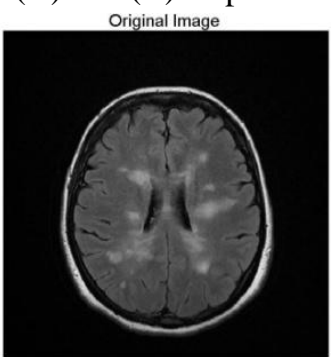

(B)
Fig. 3 T1 Weighted MRI images as original Images. (A) in sagittal plane and (B) in horizontal plan cross sections.

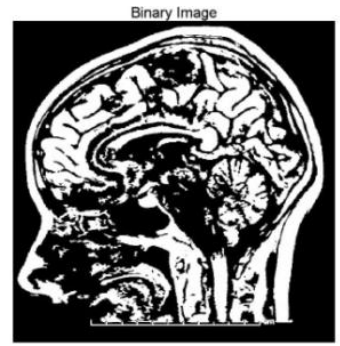

(A)

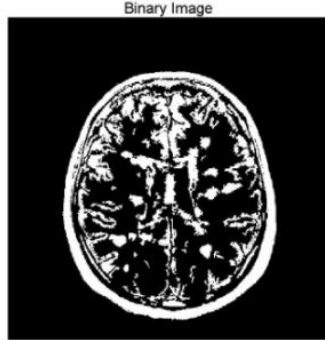

(B)
Fig. 4 Pre-processed binary images in (A) sagittal plane and $(\mathrm{B})$ horizontal plane
To demonstrate the pre-processing and binary conversions, the results in sagittal plane and horizontal plane are shown in Fig. 4 as "Binary Image". The cleaned images are shown in figure 5 . The calvarial bone segmented from the backgroung are shown in figure 6 and their inverted images in Fig. 7. Finally, the calvarial bone from water segmentation method are shown in Fig. 8.
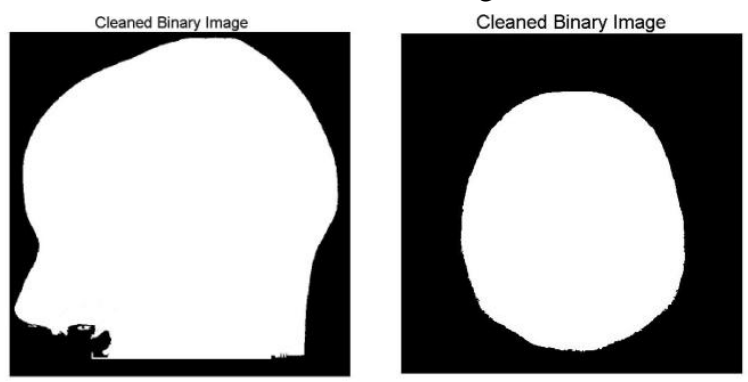

Fig. 5 Cleaned images in sagittal and horizontal planes
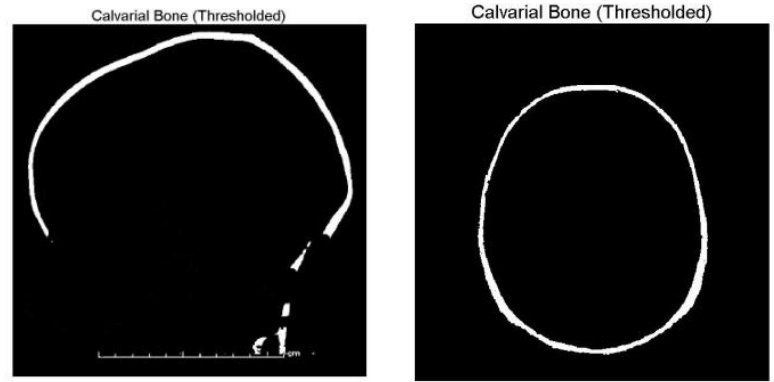

Fig. 6 Calvarial bone in sagittal and horizontal planes

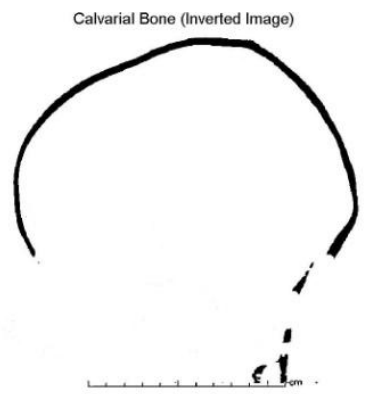

Calvarial Bone (Inverted Image

Fig. 7 Calvarial bone inverted images of above in sagittal and horizontal planes
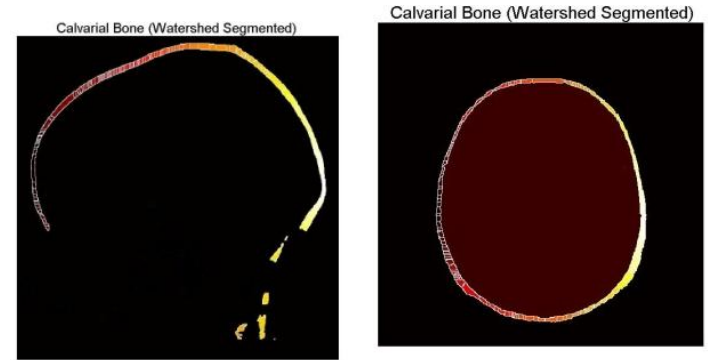

Fig. 8 Watershed segmented calvarial bone in sagittal and horizontal planes

Analysis of calvarial bone is a pathophysiological procedure which is normally done in radiographic images. Their evaluation is essential for the patients with calvarial lesions [19][20], calvarial bone fractures [21] and calvarial bone grafts [22]. In these cases the evaluation is carried out directly on MRI/CT scans. But, it would be better to 


\section{International Journal of Advanced Research in Computer and Communication Engineering}

\section{Vol. 4, Issue 12, December 2015}

do the analysis in the computer with the image processing tools. Computer based image analysis techniques are attracting researchers due to their software capabilities. The proposed image segmentation is fast and easy to implement that may help for the related diagnosis as well as post-operative patients to plan for future course of treatments. While implementing the method, care should be taken against over-segmentation which can be accomplished by selecting suitable values in the programming.

\section{CONCLUSION}

Segmentation for image analysis has its own significant imprint in medical imaging. Watershed method of segmentation is being used for various applications in digital image processing. The methodology is explained in steps and demonstrated on MR images. Segmented images of calvarial bone obtained in this work could be useful for neurosurgeons or radiologists. After scanning procedure, the image analysis can be performed as and when required. There will be scope to evaluate precisely by changing parameters for better treatment plans. It is also possible to evaluate quantitatively from the results.

\section{REFERENCES}

[1] Pal NR and Pal SK. A review on image segmentation techniques. Pattern Recogn. Vol. 26, (9), pp 1273-1294, 1993.

[2] Wankai Deng, Wei Xiao, He Deng, Jianguo Liu, "MRI Brain Tumor Segmentation With Region Growing Method Based On The Gradients And Variances Along And Inside Of The Boundary Curve", in Proc. 2010 IEEE $3^{\text {rd }}$ Int. Conf on Biomedical Engineering and Informatics, pp.393-396, 2010.

[3] Romer A. S., and Parsons, T. S.. "The Vertebrate Body. " Saunders College, Philadelphia., 1977.

[4] Tubbs, R. Shane; Bosmia, Anand N.; Cohen-Gadol, Aaron A. "The human calvaria: a review of embryology, anatomy, pathology, and molecular development". Child's Nervous System., 28 (1): 23-31, 2011 .

[5] Wecht DA, Sawaya R. Lesions of the calvaia: surgical experience with 42 patients. Ann. Surg. Oncol. 1997, 4, 28-36.

[6] Hodges F. Pathology of the skull. In. Taveras J, Ferrucci J. eds. Radiology: diagnosis, imaging and intervention. $2^{\text {nd }}$ ed. Vol. 3, Philadelphia, Lippincott, 1989, 1-21.

[7] Bastug D, Ortiz O, Sydney S. Schochet. Hemangiomas in the Calvaria: Imaging Findings. AJR; March 1995;164:683-687.

[8] Pritee Gupta, Vandana Malik and Mallika Gandhi, Implementation of Multilevel Threshold Method for Digital Images Used In Medical Image Processing, International Journal of Advanced Research in Computer Science and Software Engineering, Vol.2 Issue 2, 2012.

[9] Y.J.Zhang and J.J.Gerbrands, Objective and quantitative segmentation evaluation and comparison, Signal Processing 39, 4354 (1994).

[10] Law T, Itoh H, Seki H. Image filtering, edge detection, and edge tracing using fuzzy reasoning. IEEE Trans PAMI., 18:481-91, 1996.

[11] G. Chen, T. Hu, X. Guo, and X. Meng, "A fast region-based image segmentation based on least square method," in Proc. IEEE International Conference on Systems, Man and Cybernetics, SMC, pp.972-977, 2009.

[12] Ramesh N, Yoo JH, Sethi IK. Thresholding based on histogram approximation. IEEE Proc Vision Image Signal Proc. 1995;142:271-9.

[13] S. Zhu, X. Xia, Q. Zhang, and K. Belloulata, "An image segmentation algorithm in image processing based on threshold segmentation," in Proc. Third International IEEE Conference on Signal-Image Technologies and Internet-Based System, SITIS'O., pp. 673-678, 2007.
[14] H. Digabel and C. Lantuéjoul, "Iterative algorithms," in Proceedings of the 2nd European Symposium on Quantitative Analysis of Microstructures in Materials Sciences, Biology and Medicine, pp. 85-99, Caen, France, October 1977.

[15] L. Vincent and P. Soille, "Watersheds in digital spaces: an efficient algorithm based on immersion simulations," IEEE Transactions on Pattern Analysis and Machine Intelligence, vol. 13, no. 6, pp. 583598, 1991.

[16] L. Vincent, "Morphological grayscale reconstruction in image analysis: applications and efficient algorithms," IEEE Transactions on Image Processing, vol. 2, no. 2, pp. 176-201, 1993.

[17] Wafa Abid Fourati and Mohamed Salim Bouhlel, "Trabecular Bone Image Segmentation Using Wavelet and Marker-Controlled Watershed Transformation," Chinese Journal of Engineering, vol. 2014.

[18] Ravi S, Khan AM. Bio-Medical Image Segmentation Using Marker Controlled Watershed Algorithm: A Case Study. IJRET, Vol 3, Spl. Iss. 3, pp 26-30. May 2014.

[19] Yalçin O, Yildirim T, Kizilkiliç O, et al. CT and MRI findings in calvarial non-infectious lesions. Diagn Interv Radiol2007;13(2):6874.

[20] Lloret I, Server A, Taksdal I. Calvarial lesions: A radiological approach to diagnosis. Acta Radiol. Jun;50(5):531-42., 2009.

[21] L. Santiago Medina. Three-dimensional CT Maximum Intensity Projections of the Calvaria: A New Approach for Diagnosis of Craniosynostosis and Fractures. AJNR., 2000 (21): pp. 1951-1954.

[22] Audrey G. Sinclair, and Daniel J. Scoffings. Imaging of the Postoperative Cranium. RadioGraphics. Vol. 30 (3), March 2010 